\title{
Efeitos de uma intervenção analítico-comportamental e do treino de relaxamento em mulheres com Fibromialgia e má qualidade do sono: Um estudo piloto
}

Luziane de Fátima Kirchner

Maria de Jesus Dutra dos Reis

\section{RESUMO}

Este estudo avaliou o efeito de uma intervenção analítico-comportamental com componentes para dor, sono e relaxamento $(\mathrm{GT}, \mathrm{n}=6)$, comparado a um grupo submetido ao treino de relaxamento isolado $(G R, n=3)$ e grupo sem intervenção $(G C, n=3)$. As participantes foram mulheres (idade média 53,5 anos) com diagnóstico de Fibromialgia (FM) e queixa de má qualidade do sono. Instrumentos de autorrelato para avaliação da experiência global de dor, incapacidade relacionada à dor, qualidade do sono, ansiedade, depressão, estresse e fração livre do cortisol ao despertar, foram aplicados antes e após oito sessões, e no Follow-Up (30 dias). As participantes responderam a um questionário de satisfação ao final da intervenção. Os dados mostraram que o GT obteve mudança positiva (pós < pré) nos indicadores de incapacidade relacionada à dor e ansiedade, e o GR de experiência global de dor, com manutenção dos efeitos apenas no GT. O GR apresentou mudanças negativas (pós > pré) na resposta do cortisol e autorrelato do estresse e o GC na incapacidade relacionada à dor. Ao final do estudo, as participantes dos grupos GT e GR relataram estar satisfeitas quanto ao atendimento recebido e perceberam melhora sobre os indicadores de saúde avaliados. Conclui-se que ambas as intervenções foram efetivas, principalmente a intervenção integral.

Palavras-chaves: técnicas psicoterapêuticas; terapia de relaxamento; fibromialgia; qualidade do sono.

\section{ABSTRACT \\ Effects of a behavior analytic intervention and relaxation training in women with Fibromyalgia and poor sleep quality: A pilot study}

This study evaluated the effect of a behavior analytic intervention with components for pain, sleep and relaxation ( $T G, n=6)$, compared to groups who underwent isolated relaxation training $(R G, n=3)$ and a group without intervention, $(C G, n=3)$. The participants were women (mean age 53.5 years) with medical diagnosis of fibromyalgia (FM) and complaints of poor sleep quality. Self-report tools for evaluating the overall experience of pain, pain-related disability, sleep quality, anxiety, depression, stress, e free cortisol fraction after awakening, were administered before and after eight sessions, and in the follow-up (30 days). Participants answered a satisfaction questionnaire at the end of the intervention. Results showed that the TG achieved a positive change (post<pre) in the indicators of pain-related disability and anxiety; the same happened to the RG in the evaluation of the overall experience of pain, with maintenance of the effects only in the TG. The RG revealed negative changes (post>pre) in the cortisol responses and self-reported stress and the CG did it in pain-related disability. At the end of the study, the participants of the TG and RG reported satisfaction with the care provided and perceived improvement on the health indicators evaluated. It was concluded that both interventions were effective, especially the integral intervention.

Keywords: psychotherapeutic techniques; relaxation therapy; fibromyalgia; sleep quality.

A Fibromialgia (FM) é uma síndrome reumática caracterizada por dores músculoesqueléticas crônicas difusas e sítios dolorosos (tender points), que pode acompanhar queixas de fadiga, rigidez matinal e prejuízos na qualidade do sono (Provenza et al., 2004). Além disso,

\section{Sobre os Autores}

L.F.S.

orcid.org/0000-0002-3579-1768 Universidade Federal de São Carlos (UFSCar) - São Carlos, SP luzianefk@gmail.com

M.J.D.R.

orcid.org/0000-0002- 5128-2074 Universidade Federal de São Carlos (UFSCar) - São Carlos, SP jesus-reis@uol.com.br

\section{Direitos Autorais}

Este é um artigo de acesso aberto e pode ser reproduzido livremente, distribuído, transmitido ou modificado, por qualquer pessoa desde que usado sem fins comerciais. 0 trabalho é disponibilizado sob a licença Creative Commons CCBY-NC.

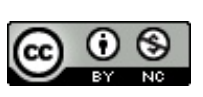




\section{-4* INTERACÃO EM LF PSICOLOGIA}

alguns estudos demonstraram alta prevalência de depressão, ansiedade (Bernik, Sampaio \& Gandarela, 2013) e possível comprometimento na atividade do eixo hipotalâmico-pituitária-adrenal (eixo HPA), sinalizando respostas de estresse crônico em mulheres com FM (Catley, Kaell, Kirschbaum \& Stone, 2000; McLean et al., 2005). Gur et al. (2002) observaram escore elevado na Escala Hamilton para depressão e níveis mais baixos de cortisol salivar no início da manhã (Cortisol Awekening Response - CAR) nas mulheres com FM e em comparação ao grupo controle saudável. Nos estudos de Riva, Mork, Westgaard, Ro e Lundberg (201) e Ross et al., (2010) níveis mais baixos na resposta do cortisol ao longo do dia, em mulheres com FM, também foram correlacionados com aumento de citocinas pró-inflamatórias, maior sensibilidade à dor, além de sintomas de fadiga, depressão e problemas no sono. Esses dados corroboram com os achados de outros estudos que apontaram a existência de fortes correlações entre variáveis clínicas (ansiedade, depressão e distúrbios do sono) e psicofisiológicas (alterações na secreção hormonal), com a presença e o comprometimento da FM (Bernik et al., 2013; Riedel, Layka, \& Neeck, 1998), embora não exista a comprovação de que essas variáveis sejam responsáveis por sua etiologia. No entanto, com base na inter-relação dessas variáveis e sua importância no quadro de FM, pesquisas têm buscado avaliar a abrangência de benefícios que diferentes abordagens terapêuticas podem proporcionar a curto e a longo prazo, e o seu custo-benefício (Wagner, Chandren, DiBonaventura, \& Cappelleri, 2013).

Intervenções comportamentais apresentaram evidências de efetividade e, portanto, constituem parte dos procedimentos clínicos padrão no tratamento da FM (Heymann, et al. 2010; Provenza et al., 2004). Existem diferentes estratégias comportamentais para o tratamento da dor crônica, dentre elas o modelo de condicionamento clássico baseado na exposição graduada de situações as quais o indivíduo evita por ter anteriormente sentido dor; o modelo operante que se baseia na relação entre os comportamentos de dor (e.g.: maior tempo em repouso, pedido de medicação, queixas relacionadas à dor, entre outros) e a sua manutenção por regras (e.g.: "sair de casa vai piorar a dor") ou pelas consequências a curto prazo fornecidas pelo ambiente social; e o modelo cognitivo que destaca o papel causal das crenças e distorções cognitivas, que podem intensificar a percepção da dor (Glombiewsk et al., 2010; Vandenberghe, 2014). Este estudo baseou-se no modelo operante para a proposta de intervenção em um dos grupos, direcionando o olhar para a análise funcional dos comportamentos de dor e problemas de sono, e as variáveis que os mantém. Comunicar-se por meio da dor é, por exemplo, um comportamento que tende a ser reforçado no ambiente social ao longo da história do indivíduo à medida que outros comportamentos adaptativos são extintos ou não

\section{Luziane de Fátima Kirchner e Maria de Jesus Dutra dos Reis}

existem em decorrência de um repertório de interação social deficitário (Fordyce, 1976). As características do ambiente físico (e.g.: carga horária de trabalho e desgaste no desempenho de tarefas diárias, alterações na rotina e nos comportamentos que antecedem o sono, ausência de atividades físicas) e social (e.g.: dificuldade do indivíduo para expor o que sente e o que é realmente importante para ele, dificuldades na organização da rotina em decorrência da necessidade de atender o outro) podem impactar nos problemas de dor e do sono e foram, portanto, o foco da análise. Utilizou-se como recurso, tanto as estratégias psicoeducativas quanto o manejo das contingências individuais (específicas a cada participante).

$\mathrm{O}$ treino de relaxamento também foi adotado como uma estratégia de intervenção. Dentre eles, o Relaxamento Muscular Progressivo de Jacobson, que visa a discriminação perceptiva dos níveis de tensão a partir dos exercícios de tensãodistensão dos grupos musculares, e o Relaxamento Autógeno de Shultz, que auxilia na ativação do sistema nervoso parassimpático responsável em diminuir as respostas de estresse (para maiores detalhes de procedimentos e aplicações dessas técnicas o leitor poderá consultar Lipp, 1997; Vera \& Villa, 2002). Essas técnicas foram descritas na década de 1930 (Rimm \& Masters 1983) e inseridas na terapia comportamental anos depois, em conjunto com o processo de dessensiblização sistemática (Wolpe, 1973). A partir da aplicação dessas técnicas a uma diversidade de problemas de saúde (Lipp, 1997) elas foram aprimoradas e reconhecidas por si só como importantes instrumentos terapêuticos. Atualmente o treino de relaxamento é descrito em conjunto com outras técnicas de intervenção, em protocolos de tratamento da dor crônica, em específico a FM (Provenza et al., 2004).

Estudos compararam os efeitos do treino de relaxamento muscular progressivo (Linton \& Götestan, 1984) e autógeno (Keel, Bodoky, Gerhard, Muller \& Comparison, 1998) aplicados isoladamente em pessoas com dor crônica e em conjunto com outras estratégias de intervenção analítico-comportamental. Os autores observaram que as intervenções em conjunto apresentaram resultados com escores mais altos e para a maior quantidade de participantes, no que se refere à redução da ingestão de medicamentos (analgésicos) e sintomas depressivos, aumento da capacidade funcional (Linton \& Götestan, 1984), melhora de problemas de sono e outros sintomas, como náusea, fraqueza, irritabilidade (Keel et al., 1998). Apesar disso, a aplicação isolada do treino de relaxamento muscular progressivo também foi capaz de melhorar a capacidade funcional e intensidade da dor, avaliada tanto por instrumento de autorrelato (Linton \& Götestan, 1984; Meeus et al., 2015; Turner, 1982) quanto por testes de estimulação mecânica de sensibilidade à dor (Coppieters et al, 2016). Os estudos sugerem a efetividade dessas intervenções a pessoas 


\section{-4* INTERACÃO EM L* PSICOLOGIA}

com FM, mas ainda não é evidente na literatura o quanto o treino de relaxamento pode ser eficaz, quando comparado a outras estratégias de intervenção analítico-comportamental. A comparação de resultados, considerando os estudos descritos acima, pode ser limitada pelas diferenças de procedimentos, medidas de avaliação e variabilidade clínica dos participantes.

Em buscas nas bases de dados do Portal Capes, no período de 2014 a 2016, não foram encontrados estudos que visassem comparar os efeitos de componentes de intervenção analítico-comportamental para dor e sono com as técnicas de relaxamento. Em estudos que se aproximaram desta problemática, ou seja, que visaram comparar o treino de relaxamento com outras estratégias de intervenção analíticocomportamental (Linton \& Götestan, 1984; Keel et al. 1998), não foram incluídas as medidas dos níveis de cortisol salivar como resposta neuroendócrina do estresse. Contudo, assim como os dados prévios demonstraram que os sintomas da FM (dor, fadiga, depressão, alterações) estão relacionados ao comprometimento no eixo HPA (Riva et al., 2010; Ross et al., 2010), mudanças nas respostas de estresse talvez ocorram se as intervenções forem capazes de reduzir os outros sintomas da FM.

Considerando os dados descritos até o momento, formulase a hipótese de que a junção de estratégias analítico-comportamentais para manejo da dor e do sono, em conjunto com o treino de relaxamento, seja mais eficaz em reduzir os sintomas clínicos, bem como as medidas diretas do estresse (cortisol salivar), em pessoas com FM. Porém, se a aplicação isolada do treino de relaxamento for apontada como eficaz nesta direção, ela poderá ser incentivada na prática clínica como uma estratégia de baixo custo e fácil aplicação (Kutz, Caudill \& Benson, 1983). Diante disso, o presente estudo avaliou o efeito de uma intervenção analítico-comportamental para dor, sono e treino de relaxamento em mulheres com FM e má qualidade do sono, comparando os efeitos isolados do treino de relaxamento e de um grupo controle (sem intervenção). Para tanto, foram consideradas as medidas de qualidade do sono, dimensão global da experiência de dor, incapacidade relacionada à dor, sintomas de ansiedade, depressão e estresse, além da resposta de cortisol ao despertar (CAR).

\section{MÉTODO}

\section{PARTICIPANTES}

A amostra foi constituída por 12 mulheres com queixa de má qualidade do sono (escore maior do que 7 no Índice de Qualidade do Sono de Pittsburg, versão brasileira - PSQI-Br) e que apresentaram diagnóstico médico de Fibromialgia.
Com o intuito de estabelecer a homogeneidade de algumas variáveis clínicas, todas as participantes também apresentavam níveis leve a moderado de ansiedade (escore entre 11 a 30 para o Inventário de Ansiedade Beck - BAI) e de depressão (escore entre 12 a 35 para o Inventário de Depressão Beck - BDI) no período inicial da coleta de dados. As participantes não estavam usando medicação para sono ou participando de tratamento psicoterapêutico no período do estudo.

Conforme disposto na Tabela 1, na totalidade da amostra, a idade média foi de 53,5 anos ( $d p \pm 6.0$ ), tendo a maioria indicado como escolaridade máxima o $2^{\circ}$ grau completo $(n=9$, $75 \%)$ e estado civil de casada $(n=9,75 \%)$. Parte substancial declarou ter uma atividade profissional externa ao lar $(n=8$, $66,6 \%$ ); sendo que nove participantes (75\%) estavam em exercício de sua função laboral, enquanto que três delas (25\%) estavam afastadas do trabalho por motivos de saúde e exerciam pouca ou nenhuma atividade em casa. Referente às condições clínicas, as participantes indicaram, predominantemente, comorbidade com outras dores musculoesqueléticas, como artrite ou artrose $(n=8,66,6 \%)$ e/ou hipertensão ( $n=4$, $33,3 \%$ ), faziam uso regular de anti-inflamatórios ( $n=8$, $66,6 \%$ ), antidepressivos ( $n=7,58.3 \%$ ) e tratamento fisioterápico $(n=6,50 \%)$ no período inicial da coleta de dados. Além disso, 11 mulheres (91,6\%), exceto $\mathrm{P} 1$, encontravam-se no período da pós-menopausa (há mais de dois anos de ausência de menstruação).

\section{LOCAL/MATERIAIS E INSTRUMENTOS}

A coleta de dados foi realizada em três unidades de atendimento localizadas em uma cidade do interior de São Paulo, que disponibilizavam de salas para atendimento individual. Duas unidades eram vinculadas a universidades, uma privada e outra pública, e a outra unidade era vinculada ao setor público de saúde. Os três locais foram disponibilizados para facilitar o acesso e a adesão das participantes. As sessões eram preferencialmente agendadas com as participantes nas datas em que elas vinham à unidade para outros atendimentos, e em caso de faltas, as sessões eram remarcadas. Além disso, algumas avaliações foram realizadas pelas participantes em suas residências.

As avaliações foram conduzidas antes (Avaliação Inicial), depois (Avaliação Final) e após 30 dias da intervenção (Follow-Up) e visaram mensurar as seguintes variáveis: qualidade do sono, dimensão global da experiência de dor, incapacidade relacionada à dor, sintomas de ansiedade, depressão, estresse e reposta do cortisol ao despertar (CAR). Para isso, instrumentos psicodiagnósticos de autorrelato foram aplicados individualmente, em uma das unidades de atendimento. Estes instrumentos foram:

Índice de Qualidade do Sono de Pittsburg, versão 


\section{- INTERACÃO EM ET PSICOLOGIA}

Tabela 1. Dados sociodemográficos e clínicos das participantes

\begin{tabular}{|c|c|c|c|c|c|c|c|}
\hline Participante & Idade & Escolaridade & $\begin{array}{l}\text { Estado } \\
\text { Civil }\end{array}$ & $\begin{array}{l}\text { Condição de } \\
\text { saúde }\end{array}$ & $\begin{array}{c}\text { Tratamentos } \\
\text { /atividades } \\
\text { atuais }\end{array}$ & $\begin{array}{c}\text { Medicação } \\
\text { em uso } \\
\text { (quantidade >1) }\end{array}$ & $\begin{array}{c}\text { Profissão/ } \\
\text { situação atual }\end{array}$ \\
\hline $\mathrm{P} 1$ & 51 & $2^{\circ} \mathrm{G} . \mathrm{C}$ & casada & $\begin{array}{l}\text { hipertensão, } \\
\text { diabetes }\end{array}$ & caminhada & $\begin{array}{c}\text { anti- } \\
\text { hipertensivos(3), } \\
\text { diurético (1), } \\
\text { antidiabético }\end{array}$ & $\begin{array}{c}\text { auxiliar de } \\
\text { enfermagem/ } \\
\text { trabalhando }\end{array}$ \\
\hline P2 & 46 & $3^{\circ} \mathrm{G} . \mathrm{C}$ & casada & $\begin{array}{l}\text { artrite, } \\
\text { artrose }\end{array}$ & $\begin{array}{c}\text { sem } \\
\text { tratamento }\end{array}$ & antiespasmódico & $\begin{array}{l}\text { enfermeira/ } \\
\text { trabalhando }\end{array}$ \\
\hline P3 & 48 & $1^{\circ} \mathrm{G} . \mathrm{C}$ & casada & $\begin{array}{l}\text { escoliose, } \\
\text { osteofitose }\end{array}$ & $\begin{array}{l}\text { sem } \\
\text { tratamento }\end{array}$ & $\begin{array}{c}\text { antidepressivo, } \\
\text { anti-inflamatório }\end{array}$ & $\begin{array}{c}\text { auxiliar de } \\
\text { limpeza/afastada }\end{array}$ \\
\hline P4 & 59 & $2^{\circ} \mathrm{G} . \mathrm{C}$ & casada & $\begin{array}{c}\text { hipertensão, } \\
\text { desvio na coluna }\end{array}$ & alongamento & $\begin{array}{l}\text { antidepressivo, } \\
\text { analgésicos (3), } \\
\text { anti-hipertensivo }\end{array}$ & $\begin{array}{c}\text { do lar/ } \\
\text { trabalhando }\end{array}$ \\
\hline P5 & 58 & $2^{\circ} \mathrm{G} . \mathrm{C}$ & casada & $\begin{array}{c}\text { diabetes, } \\
\text { artrose, } \\
\text { osteofitose }\end{array}$ & $\begin{array}{l}\text { fisioterapia, } \\
\text { caminhada }\end{array}$ & $\begin{array}{c}\text { antidiabéticos } \\
\text { (2), anti- } \\
\text { inflamatórios(2), } \\
\text { antidepressivo }\end{array}$ & $\begin{array}{c}\text { do lar/ } \\
\text { trabalhando }\end{array}$ \\
\hline P6 & 63 & $2^{\circ} \mathrm{G} . \mathrm{C}$ & solteira & $\begin{array}{c}\text { hipertensão, } \\
\text { refluxo } \\
\text { gastroesofágico, } \\
\text { artrose }\end{array}$ & fisioterapia & $\begin{array}{l}\text { anti-hipertensivo, } \\
\text { anti-ulcerosos } \\
\text { anti- } \\
\text { inflamatórios(2), } \\
\text { analgésico (2) }\end{array}$ & $\begin{array}{c}\text { cuidadora de } \\
\text { criança/ } \\
\text { afastada }\end{array}$ \\
\hline P7 & 45 & $2^{\circ} \mathrm{G} . \mathrm{C}$ & divorciada & $\begin{array}{l}\text { bursite, } \\
\text { artrite }\end{array}$ & $\begin{array}{l}\text { sem } \\
\text { tratamento }\end{array}$ & $\begin{array}{c}\text { analgésico, } \\
\text { antidepressivo, } \\
\text { anti-inflamatório }\end{array}$ & $\begin{array}{l}\text { merendeira/ } \\
\text { afastada }\end{array}$ \\
\hline P8 & 57 & $2^{\circ} \mathrm{G} . \mathrm{I}$ & casada & $\begin{array}{l}\text { artrite, } \\
\text { gastrite }\end{array}$ & fisioterapia & $\begin{array}{c}\text { corticóide, } \\
\text { antidepressivos } \\
\text { (2), anti- } \\
\text { inflamatórios (2) }\end{array}$ & $\begin{array}{c}\text { auxiliar de } \\
\text { limpeza/ } \\
\text { trabalhando }\end{array}$ \\
\hline P9 & 47 & $2^{\circ} \mathrm{G} . \mathrm{C}$ & casada & escoliose & fisioterapia & $\begin{array}{c}\text { anti-inflamatório, } \\
\text { antidepressivo }\end{array}$ & $\begin{array}{l}\text { comerciária/ } \\
\text { trabalhando }\end{array}$ \\
\hline P10 & 56 & $2^{\circ} \mathrm{G} . \mathrm{C}$ & casada & $\begin{array}{l}\text { hipertensão, } \\
\text { artrite, } \\
\text { tendinite, } \\
\text { enxaqueca }\end{array}$ & $\begin{array}{l}\text { fisioterapia, } \\
\text { caminhada, } \\
\text { hidroginástica }\end{array}$ & anti-inflamatório & $\begin{array}{c}\text { do lar/ } \\
\text { trabalhando }\end{array}$ \\
\hline P11 & 53 & $2^{\circ} \mathrm{G} . \mathrm{C}$ & casada & $\begin{array}{c}\text { gastrite, } \\
\text { refluxo } \\
\text { gastroesofágico, } \\
\text { osteofitose, } \\
\text { artrose }\end{array}$ & fisioterapia & $\begin{array}{c}\text { antiácido, } \\
\text { antidepressivo }\end{array}$ & $\begin{array}{l}\text { costureira/ } \\
\text { trabalhando }\end{array}$ \\
\hline P12 & 59 & $2^{\circ} \mathrm{G} . \mathrm{C}$ & solteira & $\begin{array}{c}\text { gastrite, } \\
\text { refluxo } \\
\text { gastroesofágico }\end{array}$ & $\begin{array}{l}\text { sem } \\
\text { tratamento }\end{array}$ & $\begin{array}{c}\text { anti-inflamatório, } \\
\text { antiácido }\end{array}$ & $\begin{array}{c}\text { do lar/ } \\
\text { trabalhando }\end{array}$ \\
\hline
\end{tabular}

Legenda: G.C. - Grau Completo; G.I. - Grau Incompleto. 
brasileira - PSQI-Br (Bertolazzi, 2008): é um instrumento de autorrelato que avalia a qualidade do sono no último mês, por meio de 19 itens distribuídos em sete componentes (qualidade do sono, latência do sono, duração do sono, eficiência do sono, alterações do sono, uso de medicações para o sono, e disfunção diurna), pontuados em uma escala de 0 a 3. 0 somatório dos componentes indica a pontuação global, que varia de 0 a 21. Pontuações entre 0 e 4 sugerem boa qualidade do sono, de 5 a 10 indicam qualidade de sono ruim, e acima de 10 pontos indicam a presença de distúrbio do sono. Consistência interna do instrumento $a=0,82$.

Short-form McGill Pain Questionnaire - SF-MPQ (Melzack, 1987): é um instrumento multidimensional que avalia a experiência dolorosa por meio de palavras (descritores) que o paciente escolhe para expressar a sua dor. Os descritores são divididos em três dimensões (sensorial, afetiva e avaliativa), e o participante deve indicar, dentre 15 grupos de adjetivos, aqueles que melhor descrevem a sua dor em cada dimensão. Para cada adjetivo escolhido pontua-se: 0 - nenhuma dor; 1 - dor leve; 2 - dor moderada; ou 3 - dor severa. 0 somatório da pontuação nos itens resulta no Índice de estimativa da dor - PRI Total, cuja pontuação varia de 0 a 45 . Este escore indica que quanto maior a pontuação, pior será a percepção da experiência dolorosa da participante. Os dados também podem ser avaliados separando-se as dimensões sensorial e afetiva da dor. Consistência interna do instrumento $a=0,75$.

Questionário de Impacto da Fibromialgia, versão brasileira - FIQ (Marques et al., 2006): instrumento constituído por 19 questões que investigam a incapacidade frente à dor, a partir das dimensões: capacidade funcional, situação ocupacional, distúrbios psicológicos e sintomas físicos. Atribui-se a pontuação de acordo com o somatório dos itens e divisão, da seguinte maneira: os itens para a escala 0-3 são somados e divididos por 30 , os itens para a escala $0-7$ são somados e divididos por 7, e as pontuações nas escalas analógicas são somadas divididas por 10 . O valor máximo é 100 , e quanto mais próximo a este valor, maior a percepção de incapacidade frente à dor. Consistência interna do instrumento $a=0,89$.

Inventário de Ansiedade Beck - BAI (Cunha, 2001): apresenta uma lista de 21 sintomas de ansiedade a serem avaliados pelo participante como tendo ocorrido na última semana. Cada item apresenta quatro possíveis respostas e suas respectivas pontuações: 0) absolutamente não; 1) levemente - não me incomodou muito; 2) moderadamente foi desagradável, mas pude suportar; e 3) gravemente - quase não suportei. 0 somatório dos itens a partir dessas pontuações indicará um dos quatro diferentes níveis de ansiedade, do mais leve ao de maior cuidado: Mínimo (0 a
10), Leve (11 a 19), Moderado (20 a 30) e Grave (31 a 63). Consistência interna do instrumento $a=0,88$.

Inventário de Depressão Beck - BDI (Cunha, 2001): é composto por 21 grupos de afirmativas que devem ser identificadas pelo participante como autodescritivas de aspectos do seu comportamento, seus sentimentos ou suas emoções, na última semana. Cada item apresenta quatro possíveis respostas, que devem ser pontuadas entre 0 a 3 . 0 somatório de itens a partir desta pontuação indicará um dos quatro diferentes níveis de depressão, sendo do menor para o maior: Mínimo (0 a 11), Leve (12 a 19), Moderado (20 a 35) e Grave (36 a 63). Consistência interna do instrumento $a=0,81$.

Inventário de Sintomas de Estresse para Adultos de Lipp - ISSL (Lipp, 2000): disponibiliza três quadros que solicitam a autoidentificação da ocorrência de sintomas de estresse físicos e psicológicos - no dia anterior à avaliação, na semana anterior ou no último mês. Quatro diferentes fases de estresse (Alerta, Resistência, Quase-exaustão e Exaustão) são identificáveis a partir do somatório de itens e outros critérios de correção do instrumento apresentados no manual. As três últimas fases indicam a necessidade de cuidado em saúde mental. Consistência interna do instrumento $a=0,91$.

Os níveis de cortisol foram avaliados por amostras de saliva, que oferecem uma medida da fração livre do hormônio. A avaliação da resposta do cortisol ao despertar (CAR), foi feita em dois momentos na casa das participantes, um ao despertar e outro 30 minutos após. As participantes foram esclarecidas verbalmente e por escrito a respeito de alguns cuidados (não ingerir medicamentos, alimentos, bebidas, fumar, escovar os dentes ou fazer atividade física antes de cada coleta, além de não realizar a coleta se apresentar sangramento bucal, e anotar o horário de realização de cada coleta). Caso não fosse possível cumprilos no dia estipulado, a participante era orientada a realizar a coleta no dia seguinte. As amostras de saliva (500 $\mu \mathrm{l} \mathrm{cada)}$ foram coletadas em tubos de polipropileno (Eppendorf $\AA$, Hamburgo, Alemanha), entregues aos pesquisadores no mesmo dia da coleta, e armazenadas em freezer a $-20^{\circ} \mathrm{C}$. Para análise em laboratório de pesquisa, as amostras foram descongeladas e analisadas por meio de ensaio imunoenzimático (Cortisol ELISA ${ }^{\circ}$, IBL International, Hamburgo - Alemanha). Não foi possível determinar um valor de referência do cortisol ao despertar (CAR) para análise, pelo fato de as participantes realizarem a primeira coleta da manhã em horários diferentes. Mas como em indivíduos saudáveis ocorre um pico nos níveis de cortisol de 20 a 30 minutos após o despertar (Riva et al., 2010), foi considerado, neste estudo, "resposta positiva" se o nível do cortisol na segunda coleta fosse $40 \%$ maior que o nível do 


\section{- INTERACÃO EM ET PSICOLOGIA}

cortisol na primeira coleta (ao despertar), "resposta estável" se a diferença entre a primeira e a segunda coleta fosse entre 59 a $0 \%$, e "resposta negativa" se a diferença entre a primeira e a segunda coleta resultasse em valor negativo. $O$ cálculo utilizado para avaliar a diferença entre a primeira coleta - A (realizada ao despertar) e a segunda coleta - B (30 minutos após) foi $=((B-A) / A * 100)$.

$\mathrm{Na}$ etapa de Avaliação Final, além dos instrumentos descritos acima, foi aplicado a todas as participantes um questionário para avaliar o nível de satisfação e a percepção de melhora de saúde da participante com relação aos procedimentos aplicados. 0 questionário foi elaborado pelos autores para os fins da pesquisa e foi composto por seis perguntas em escala formato Likert ( 0 a 5 ) e duas perguntas abertas (o que mais gostou e o que menos gostou em relação aos procedimentos).

\section{PROCEDIMENTO DE COLETA DOS DADOS}

Após aprovação do Comitê de Ética em Pesquisa do Centro Universitário Central Paulista (CAEE 32623314.6.0000.5380) e obtenção do consentimento por escrito das participantes (Termo de Consentimento Livre e Esclarecido - TCLE) foi conduzida a triagem que verificou os critérios de inclusão. Neste momento, as participantes que apresentavam tais critérios foram orientadas a respeito dos procedimentos do estudo, da importância de manter a dose, tipo de medicação e outros tratamentos que já faziam uso durante todo o período de coleta de dados e da importância de não faltar às sessões. As que concordaram com os procedimentos foram alocadas em um dos três grupos, pareando-se as caraterísticas clínicas a partir dos seguintes critérios: níveis de ansiedade e depressão, interesse e disponibilidade para participar de intervenções. As participantes P1, P2 e P3 foram alocadas no Grupo Controle (GC) e apenas responderam as avaliações, no período em que eram designadas. As participantes P4, P5 e P6 constituíram o Grupo Relaxamento (GR) e foram individualmente submetidas a oito sessões, com periodicidade semanal e duração de 30 minutos cada, distribuídas em: a) esclarecimento de dúvidas sobre o relaxamento realizado em casa (10 minutos); b) treino de relaxamento (20 minutos). As intervenções programadas para relaxamento foram: sessão 1) informações sobre os benefícios do treino de relaxamento ao organismo, explicação e treino de respiração diafragmática - as participantes foram solicitadas a inspirar em 3 segundos pelo nariz, observando o movimento de expansão do abdômen e, na sequência, a expirar lentamente (em 6 segundos) pela boca - este treino foi realizado até que a participante conseguisse realizar o movimento de expansão abdominal ao inspirar, ao invés do movimento torácico; sessão 2) treino de Relaxamento Muscular Progressivo e Autógeno e entrega do
CD de relaxamento (gravado em estúdio com base nas instruções de Vera \& Vila, 2002), para que a participante pudesse realizá-lo em casa; sessões 3 a 8) treino de Relaxamento Muscular Progressivo e Autógeno, conforme disposto em Vera e Vila (2002). E, por fim, as participantes P7 a P12 foram alocadas no Grupo Tratamento (GT), cujos procedimentos de intervenção foram oito sessões individuais, com periodicidade semanal e duração de 1 hora e 30 minutos cada, estruturadas da seguinte maneira: a) acolhimento e esclarecimento de dúvidas sobre os procedimentos de intervenção (30 minutos); b) atividade temática relacionada ao manejo da dor ou do sono (40 minutos); e, c) treino de Relaxamento Muscular Progressivo e Autógeno - similar às sessões 1 a 8 aplicadas ao GR (20 minutos). Em quatro sessões foram abordados assuntos específicos para manejo da dor, tais como: 1) psicoeducação referente ao diagnóstico de FM e tratamentos possíveis, relação dor e emoção, 2) identificação do ambiente físico/social de cada participante que apresentam relação com a dor, a partir de dados obtidos na avaliação inicial, 3) psicoeducação referente a condições do ambiente físico que interferem na dor e manejo de tais contingências para melhorar capacidade funcional, e 4) psicoeducação referente a assertividade e manejo de contingências do ambiente social para lidar assertivamente com os aspectos deste ambiente que interferem na dor. Nas outras quatro sessões foram abordadas temáticas para manejo do sono, tais como: 1) psicoeducação referente a importância do sono e de cada fase do sono, fatores que podem interferir na qualidade do sono e tratamentos possíveis, 2) identificação de estímulos do ambiente físico/ social de cada participante que interferem na qualidade do sono, a partir de dados obtidos na avaliação inicial; 3) psicoeducação referente aos comportamentos diurnos e sua interferência na qualidade do sono (e.g.: quantidade e duração de cochilos ao dia, duração do tempo em repouso e de atividades realizadas no quarto) e manejo de contingências do ambiente direcionada à instalação e manutenção dos comportamentos diurnos em benefício da qualidade do sono; 4) psicoeducação referente aos comportamentos que antecedem o horário de dormir e seus efeitos na qualidade do sono (e.g.: alimentação, atividades sociais e ocupacionais) e manejo de contingências referentes a tais comportamentos capazes de promover melhora na qualidade do sono. Ao final da coleta de dados as participantes receberam devolutivas individuais e aquelas que não identificaram melhora em relação às variáveis observadas foram encaminhadas para atendimentos individuais e voluntários no Serviço Escola de Psicologia de uma das universidades participantes.

As sessões dos grupos GR e GT foram gravadas em áudio digital (MP4) e analisadas por observadores independentes. 


\section{H. INTERACÃO EM LF PSICOLOGIA}

Após uma hora de treinamento com dois observadores, alunos do quarto ano de graduação em Psicologia, estes realizaram separadamente as avaliações de sessões sorteadas: quatro sessões eram de manejo da dor incluindo o treino de relaxamento, quatro sessões eram de manejo do sono incluindo o treino de relaxamento, e duas sessões eram somente treino de relaxamento. 0 período de observação para cada sessão foi de 30 minutos, divididos entre dez minutos iniciais, dez minutos intermediários e dez minutos finais, com sessões e horários de início e término iguais para os observadores. Foi atribuído "concordância" a cada item da

Tabela 2. Porcentagem dos niveis de concordância entre observadores referente à estrutura, temática geral e temática especifica das sessões.

\begin{tabular}{|c|c|c|}
\hline & GR & GR \\
\hline Estrutura das sessões (acolhimento, atividade temática, relaxamento) & 100 & 83,3 \\
\hline Temáticas gerais (dor, sono ou relaxamento) & 100 & 100 \\
\hline \multicolumn{3}{|l|}{ Temáticas específicas do GT } \\
\hline \multicolumn{3}{|l|}{ Dor } \\
\hline 1) Informações sobre o diagnóstico de FM e tratamentos possíveis, relação dor e emoção; & & 100 \\
\hline 2) Identificação de fatores do ambiente físico/social que apresentam relação com a dor; & & 66,6 \\
\hline 3) Planejamento de atividades para melhorar capacidade funcional; & & 66,6 \\
\hline 4) Treino assertivo; & & 100 \\
\hline \multicolumn{3}{|l|}{ Sono } \\
\hline 1) Informação sobre a importância do sono e de cada fase do sono, fatores intervenientes; & & 100 \\
\hline 2) Relatos/identificação de hábitos diários prejudiciais a qualidade do sono da participante; & & 83,3 \\
\hline 3) Discussão sobre mudança de comportamentos diurnos para melhorar a qualidade do sono; & & 83,3 \\
\hline 4) Discussão sobre mudança de comportamentos a noite para melhorar a qualidade do sono; & & 66,6 \\
\hline
\end{tabular}

\section{ANÁLISE DOS DADOS}

Os dados para as variáveis "avaliação da experiência global de dor, qualidade do sono, incapacidade relacionada à dor, sintomas de ansiedade e depressão" foram expressos considerando o percentual da diferença entre as avaliações Inicial - Final. Ao considerar que os instrumentos para as variáveis descritas acima atribuem valores negativos (quanto melhor, mais baixo o escore), estabeleceu-se a classificação "mudança positiva" quando os escores da avaliação final foram menores que a avaliação inicial, o que pode refletir a melhora dos sintomas, e "mudança negativa" quando os escores da avaliação final foram maiores que a avaliação inicial, o que reflete na piora dos sintomas. Considerando a variabilidade clínica das participantes, uso de medicações e outros tratamentos, optou-se por apresentar também os resultados individuais para as variáveis descritas acima, além das medidas do estresse (ISSL e CAR). Análises estatísticas inferenciais não puderam ser realizadas pela quantidade de estrutura, temática geral e temática específica (no caso das sessões do grupo GT), assinalado de forma correspondente a análise feita pela pesquisadora. Na sequência, realizou-se o cálculo de concordância (concordância/(concordância + discordância)*100) entre os observadores, e entre cada observador e a pesquisadora, e se estabeleceu a média para cada item avaliado. Conforme mostrado na Tabela 2, o nível de concordância de cada item variou entre 66,6 a 100\% e que pode ser classificado, segundo o critério de Landis e Koch (1977), como concordância substancial (61 a 80) e quase perfeita (81 a 100). 
experiência de dor (SF-MPQ), incapacidade relacionada à dor (FIQ), ansiedade (BAI) e depressão (BDI). Os valores abaixo de zero denotam "mudanças positivas" após a intervenção, e os valores acima de zero denotam as "mudanças negativas".

O grupo submetido a tratamento integral (GT) apresentou mudanças positivas para todos os indicadores de saúde apresentados no gráfico, indicando que houve uma queda para todos eles, enquanto o grupo submetido ao treino de relaxamento isolado (GR) destacou-se por apresentar mudança positiva na avaliação global da experiência de dor (SF-MPQ) e ansiedade (BAI). Entretanto, nos grupos GR e GC foram observadas mudanças negativas sobre a incapacidade relacionada à dor (FIQ), e no GR a mudança negativa também foi observada para os sintomas de qualidade do sono (PSQI$\mathrm{Br})$ e depressão (BDI).

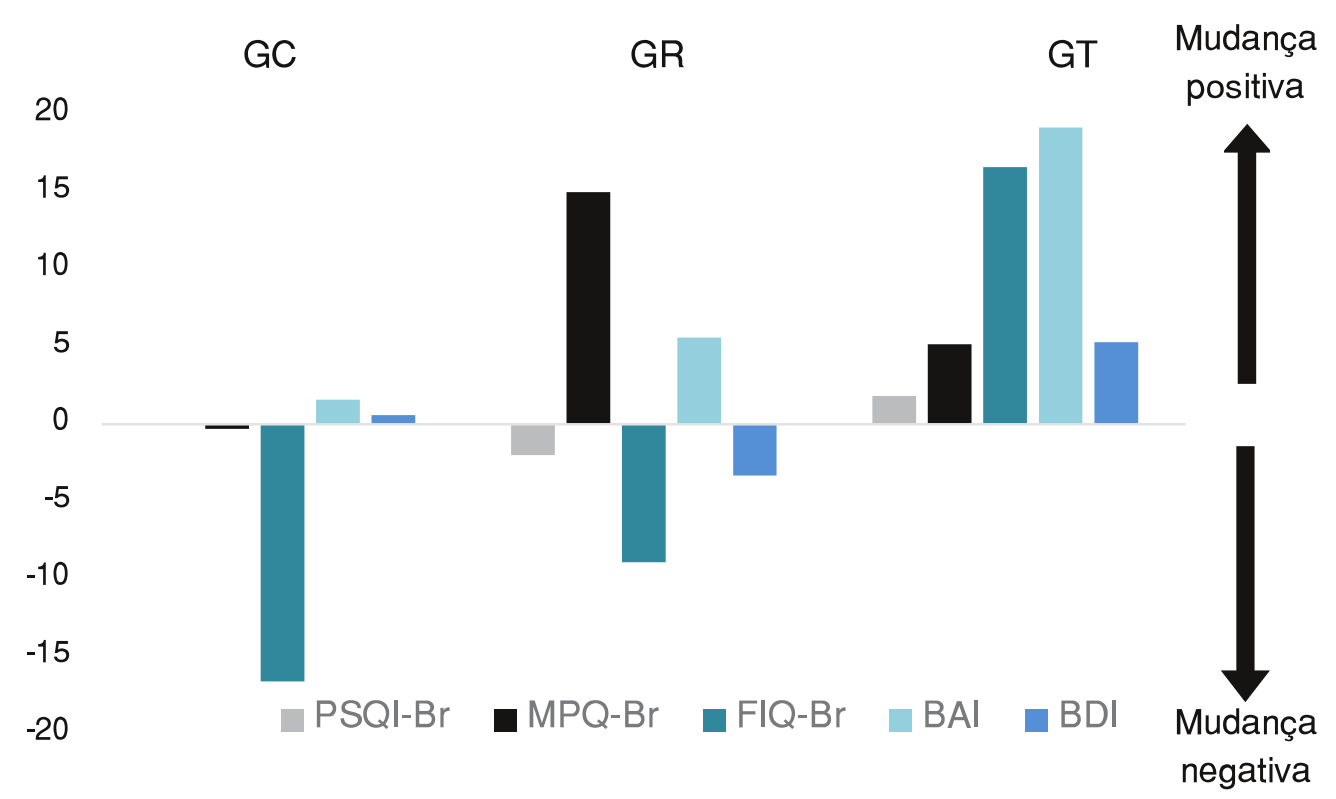

Figura 1. Percentual da diferença entre as médias das avaliações Inicial-Final nas diferentes medidas realizadas, para os diferentes grupos (GC=Grupo Controle; GR=Grupo Relaxamento; GT=Grupo Tratamento)

Na Tabela 3 são apresentados os dados individuais para as mesmas variáveis descritas acima, referindo os instrumentos cujos escores apontaram mudança positiva (indicativo de melhora considerado pela redução de $40 \%$ ou mais no escore final quando comparado ao escore inicial) e mudança negativa (indicativo de piora considerado pelo aumento de $40 \%$ ou mais no escore final quando comparado ao escore inicial), após a intervenção. Adicionalmente, foram identificadas as medidas do estresse, cujas participantes apresentaram mudanças positivas ou negativas de fase do estresse (ISSL) e de resposta do cortisol (CAR). Respostas estáveis na avaliação inicial que passaram a ser positivas na avaliação final, ou respostas negativas que passaram a ser estáveis, foram atribuídas, por exemplo, como mudanças positivas de resposta do cortisol. Os dados das participantes que se mantiveram na mesma fase do estresse e com resposta estável do cortisol não foram identificados na tabela abaixo.

Todas as participantes do GT apresentaram mudanças positivas, com redução dos sintomas de ansiedade (BAI), quatro das seis participantes (P7, P8, P9 e P12) apontaram redução da incapacidade relacionada à dor (FIQ), e a metade (três participantes) apresentou redução dos sintomas do estresse com mudança de fase (ISSL). No GR, duas das três participantes apresentaram mudança positiva na avaliação global da experiência de dor (SF-MPQ), porém, esta mesma quantidade de participantes apresentou mudança negativa para as medidas de estresse (ISSL e CAR).

Duas participantes do grupo controle, submetidas apenas às avaliações (GC), apresentaram mudança positiva para a medida de autorrelato do estresse (ISSL), porém nenhuma participante apresentou mudanças positivas nas outras medidas avaliadas, que pudessem indicar percentual de variação igual ou superior a $40 \%$. Em contrapartida, foram observadas mudanças negativas sobre incapacidade relacionada à dor (FIQ). Mudanças negativas também foram observadas para a variável qualidade do sono (PSQI-Br), nos dois grupos submetidos a intervenção, GR e GT.

As avaliações conduzidas 30 dias após a intervenção (Follow-up) permitiram verificar que as mudanças positivas, 


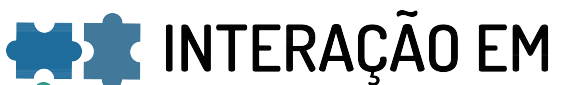 PSICOLOGIA}

Tabela 3. Identificação das medidas que apresentaram mudança positiva e negativa entre as avaliações Inicial-Final para cada participante

\begin{tabular}{cccc}
\hline Grupos/ Participantes & \multicolumn{2}{c}{$\begin{array}{c}\text { Avaliação Inicial } \mathbf{X} \text { Avaliação Final } \\
\text { Mudanças positivas }\end{array}$} & FIQ, CAR \\
\hline GC & P1 & ISSL & FIQ, ISSL \\
& P2 & - & SF-MPQ \\
P3 & ISSL & ISSL, CAR \\
& P4 & & CAR \\
& P5 & BAI & PSQI-Br, FIQ, ISSL, BDI \\
& P6 & SF-MPQ & \\
& P7 & SF-MPQ & CAR \\
& P8 & BAI, FIQ, CAR & - \\
& P9 & BAI, FIQ, SF-MPQ, BDI & SF-MPQ, CAR \\
& P10 & BAI, FIQ, ISSL, CAR & PSQI-Br \\
& P11 & BAI, ISSL & PSQI-Br, ISSL \\
\hline
\end{tabular}

GC - Grupo Controle; GR - Grupo Relaxamento; GT - Grupo Tratamento.

ISSL - Inventário de Sintomas de Estresse de Lipp; FIQ - Questionário de Impacto da Fibromialgia; CAR - Cortisol Awekening Response; SF-MPQ - Short-form McGill Pain Questionnaire; BAI - Inventário de Ansiedade Beck; PSQI-Br Índice de Qualidade do Sono de Pittsburg; BDI - Inventário de Depressão Beck;

observadas de acordo com os critérios atribuídos neste estudo, foram mantidas apenas no GT, nas medidas de ansiedade (BAI) em cinco de seis participantes, depressão, incapacidade relacionada à dor e estresse (ISSL e CAR) em uma de seis participantes. Adicionalmente, mudanças negativas, que denotariam percepção de piora dos sintomas no período de 30 dias após a intervenção, não foram observadas para nenhum dos grupos.

Foi também avaliado o nível de satisfação das participantes com relação aos procedimentos aos quais elas foram submetidas, bem como a percepção delas em relação à melhora da qualidade do sono, dor, ansiedade, depressão. Os dados seguem apresentados na Figura 2.

Conforme observado na Figura 2, as participantes dos grupos GT e GR relataram estar satisfeitas (nível de satisfação entre três e cinco, a partir de uma escala formato Likert de cinco pontos) quanto ao atendimento recebido e perceberam melhora nos indicadores de saúde avaliados,

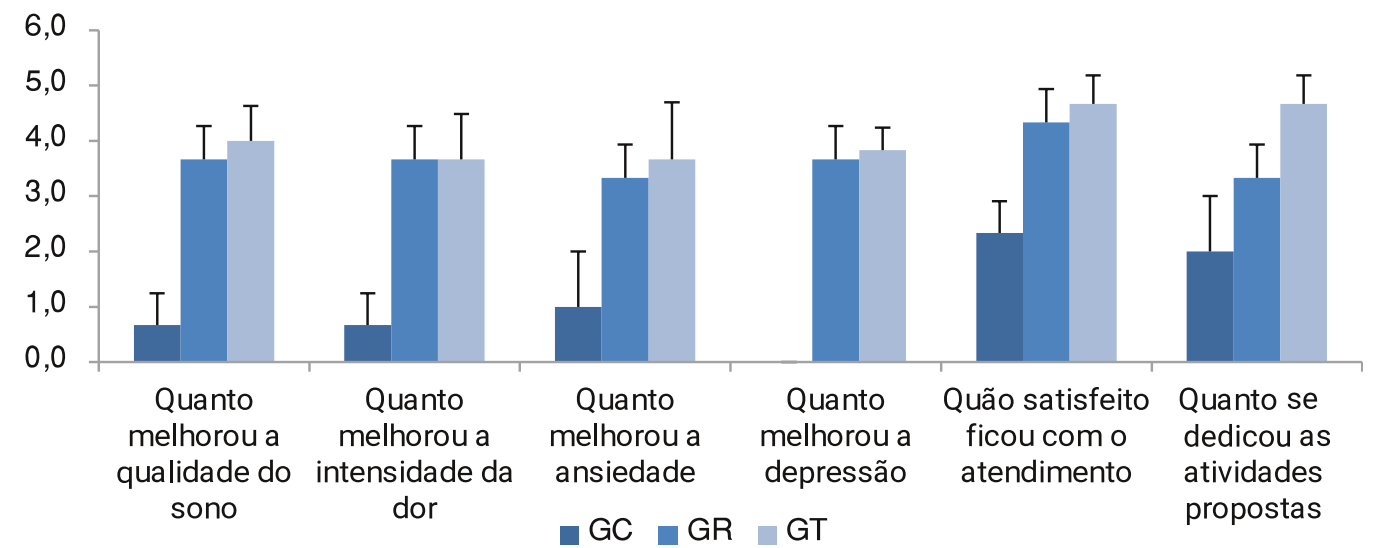

Figura 2. Média e Desvio Padrão do nível de satisfação e percepção de melhora em relação ao procedimento de coleta dos dados para os três grupos 


\section{MI* INTERACÃO EM LF PSICOLOGIA}

inclusive na qualidade do sono. Porém, para todas as questões, exceto em relação à intensidade da dor, o nível de satisfação e percepção de melhora foi maior para o grupo submetido ao tratamento integral (GT). Destaca-se que as participantes deste grupo também relataram ter se dedicado às atividades propostas em sessão mais do que as participantes dos outros grupos. Para as questões abertas, cinco participantes do grupo GT relataram "ter gostado da relação estabelecida com a terapeuta em sessão", e duas participantes do GR relataram que "gostariam de participar de outras atividades além do treino de relaxamento". Outros aspectos mencionados como negativos se referiram à distância da residência a unidade de atendimento (P3), à necessidade de realizar as avaliações em casa (P6 e P8), e a estrutura do local de atendimento (P12).

\section{DISCUSSÃO}

O objetivo deste estudo foi avaliar o efeito de uma intervenção analítico-comportamental para dor e sono com treino de relaxamento a mulheres com FM e má qualidade do sono (GT), e o efeito isolado do treino de relaxamento (GR), ambos em comparação ao grupo não submetido à intervenção (GC). No que se refere ao efeito das intervenções, a análise da média denotou que o GT apresentou mudança positiva em todos os indicadores clínicos autorrelatados, e todas as participantes deste grupo apresentaram mudança positiva em pelo menos dois desses indicadores na avaliação logo após a intervenção. Esses dados concordam com os argumentos apresentados na Conferência do National Institutes of Health Technology (NIH Technology Assessment Panel, 1996), de que a intervenção para dor e sono, aliando técnicas de relaxamento, é eficaz na redução dos sintomas de mulheres com FM.

Os indicadores que apresentaram mudança positiva para a maioria das participantes do GT foram ansiedade e incapacidade relacionada à dor, enquanto que no GR a mudança positiva foi observada principalmente para avaliação da experiência global de dor. Em literatura é apontado que o objetivo das estratégias analíticocomportamentais não é, prioritariamente, reduzir a intensidade da dor, mas substituir os comportamentos de dor estabelecidos e mantidos no ambiente para outros mais adaptativos, relacionados à melhora da qualidade de vida $\mathrm{e}$ aumento da capacidade funcional (Fordyce, 1976; Wegener, Wolfman, \& Haythornthwaite, 2011). Intervenções como esta podem trazer efeitos mais duradouros à medida que respostas adaptativas vão sendo emitidas e reforçadas no ambiente, reduzindo contingências aversivas e geradoras de ansiedade (Lundervold, Talley \& Buermann, 2006). Assim, os resultados apresentados condizem com a descrição da literatura, ao apontar os efeitos benéficos na ansiedade e incapacidade relacionada à dor para o grupo submetido à intervenção integral (GT), que se mantiveram na avaliação de Follow-up.

O treino de relaxamento visa possibilitar a discriminação dos níveis de tensão e controle do sistema nervoso parassimpático (Vera \& Vila, 2002). Nesses termos, é esperado que o treino de relaxamento apresente resultados sobre respostas fisiológicas de estresse, ansiedade e percepção do limiar de dor (Wegener et al., 2011), o que é diferente de um outro conjunto de estratégias analíticocomportamentais direcionadas a modificar respostas mais complexas. 0 resultado na redução da "experiência global de dor", observados no GR, são justificados com esta literatura (Wegener et al., 2011). Os efeitos positivos no GR em relação à dor foram observados ao final da coleta de dados, mas não se mantiveram na avaliação de Follow-Up. A manutenção dos efeitos do Relaxamento Muscular Progressivo também não foi observada no estudo de Turner (1982), em comparação a uma intervenção cognitivo-comportamental; ao invés disso, em longo prazo houve uma piora na percepção da dor para o grupo submetido somente ao relaxamento. Estes dados apoiam o argumento de que o relaxamento é eficaz na redução de respostas fisiológicas imediatas, porém a manutenção dos seus efeitos requer continuidade no treino (Turner, 1982; Vera \& Villa, 2002).

O GC apresentou mudança positiva para a medida de autorrelato do estresse (ISSL), que pode ter ocorrido por condições não controladas neste estudo (e.g.: alterações de atividades e/ou tratamento das participantes). Apesar disso, as mudanças observadas neste grupo foram principalmente negativas, com destaque para a incapacidade relacionada à dor (FIQ), o que sugere que, com o passar do tempo e a ausência de cuidados, o comprometimento relacionado à dor pode piorar. Tal argumento também é apresentado no estudo de Salvetti, Pimenta, Braga e Corrêa (2012).

Com relação ao grupo submetido ao treino de relaxamento isolado (GR), apesar dos efeitos observados na "experiência global da dor", as três participantes deste grupo também apresentaram mudanças negativas em medidas diretas ou de autorrelato do estresse (CAR e ISSL), ou seja, denotou-se o aumento de sintomas de estresse com mudança de fase e redução do nível do cortisol na segunda avaliação em comparação à primeira. Dada a importância do treino do relaxamento como uma estratégia de manejo do estresse (Lipp, 2000), era esperada a melhora deste indicador de saúde neste grupo. Uma análise individual dos dados apontou, no entanto, que as participantes deste grupo que relataram piora relacionada à percepção dos sintomas do estresse (P4 e P6) também apresentaram níveis moderados 


\section{- INTERACÃO EM ET PSICOLOGIA}

de ansiedade e depressão e alto nível de incapacidade relacionada à dor na avaliação inicial, com pouca mudança nestes indicadores ao final da intervenção. Nestes termos, é possível hipotetizar que, embora o treino de relaxamento possa ser eficaz na redução de respostas fisiológicas (Wegener et al., 2011), a presença de maior comprometimento na ansiedade, depressão e incapacidade relacionada a dor pode fazer com que esta intervenção não tenha sido suficiente para reduzir os sintomas do estresse e existe a possibilidade de ter levado a percepção de piora destes sintomas. Diversos autores concordam ao apontar que a percepção de maior gravidade das comorbidades psiquiátricas (ansiedade, depressão) pode impactar na percepção dos sintomas da FM, incluindo as respostas de estresse (Thieme \& Turk, 2005; Van Houdenhove \& Luyten, 2006).

Ao final da intervenção, P4 e P5 (do Grupo Relaxamento) apontaram mudança negativa do nível do cortisol, e apenas duas participantes, em toda a amostra, apresentaram mudança positiva nesta medida. McLean et al. (2005) destacam que pelo fato do sistema HPA estar comprometido em pessoas com FM, intervenções breves podem ser pouco efetivas para alterar tal resposta. Apenas um estudo foi encontrado (Menzies, Lyon, Elswick, Mccain \& Gray, 2014), que avaliou o estresse, por meio de medida direta (biomarcadores imunes) e de autorrelato (Perceived Stress Scale - PSS), em mulheres com FM após um treino de relaxamento. Os autores conduziram um treino de relaxamento específico (imaginação guiada, 10 semanas) e observaram efeitos positivos quando o estresse foi avaliado por medida de autorrelato, mas não por medida direta. No estudo de Catley et al. (2000), a medida direta (nível do cortisol) em pessoas com FM não apresentou alteração diante de eventos estressores agudos (situações do cotidiano), e no estudo de McLean et al. (2005) a variação desta resposta foi associada a histórias de eventos estressores ao longo da vida, como o histórico de abuso físico e sexual. Esses argumentos vão de encontro ao resultado deste estudo e é possível que a quantidade limitada de sessões (oito), aliado a uma longa exposição a eventos estressores com comprometimento no eixo HPA (Catley et al., 2000; McLean et al., 2005; Van Houdenhove \& Luyten, 2006) justifique o fato das participantes não apresentarem mudanças positivas na avaliação do cortisol (CAR). No entanto, como afirmam Catley et al. (2000) e McLean et al. (2005), os dados de pesquisas são inconsistentes em relação à resposta do cortisol em pessoas com $\mathrm{FM}$, o que requer maior investigação.

A qualidade do sono, de acordo com o escore geral do instrumento PSQI-Br, não apresentou mudança positiva nos grupos, e nem mesmo àquele submetido às quatro sessões destinadas a melhorar este aspecto (GT). Uma análise detalhada dos itens deste instrumento (PSQI-Br) revelou, no entanto, que cinco de seis participantes do GT apresentaram mudanças positivas em itens específicos: quatro participantes relataram que houve redução da quantidade de vezes por semana que demoravam mais que trinta minutos para pegar no sono, três participantes relataram reduzir a quantidade de vezes que precisavam levantar para ir ao banheiro ao longo da noite, e duas participantes relataram mudança positiva em um item especifico de qualidade do sono deste instrumento "De modo geral, como você avalia a sua qualidade do sono?". Também foram observadas mudanças positivas neste item em específico, para uma das participantes de cada um dos grupos (P2 do GC e P4 do GR). A média obtida para o Questionário de satisfação indicou que a percepção de melhora para a qualidade do sono foi positiva e similar aos outros indicadores de saúde avaliados neste instrumento. Tomadas em conjunto, essas mudanças sugerem que a intervenção tenha impactado na qualidade e nos padrões do sono, mas a quantidade de sessões talvez não tenha sido suficiente para produzir mudanças no escore geral do PSQI-Br. Estudos que aplicaram um pacote de quatro sessões da Terapia Cognitivo-Comportamental para o manejo da dor e/ou do sono a pessoas com dor crônica (Tang, Goodchild, \& Salkovskis, 2012; Vitiello, et al., 2013) apresentaram melhora na qualidade e nos padrões do sono, o que apoia os dados do presente estudo. Apesar disso, Perlis et al. (2005) argumentam que os efeitos nos indicadores de sono, para este tipo de intervenção, poderão ser observados a partir da quinta sessão. Novos estudos precisam ser conduzidos com um número maior de sessões, a fim de esclarecer tais efeitos. Sugere-se ainda que outras medidas para avaliação da qualidade do sono possam ser acrescentadas em futuros estudos, assim como instrumentos que permitam avaliar outras características do sono (e.g.: número de vezes que acorda e o tempo que passa na cama acordado ao longo da noite). É também importante considerar que o comprometimento na qualidade do sono pode ter sido impactado pela presença de outros problemas de saúde que não foram identificados no presente estudo, tais como a presença de distúrbios do sono (e.g: Síndrome da Apnéia Obstrutiva do Sono, Síndrome das Pernas Inquietas) ou sintomas do climatério decorrentes da pós-menopausa (Krakow, 2006).

Os efeitos da interação entre maturação-seleção, referido como as características das participantes que as tornam suscetíveis a responder diferencialmente ao mesmo tratamento (Campbell \& Stanley, 1979), talvez possam explicar discrepâncias nos resultados das participantes do mesmo grupo. Além de aspectos clínicos e sociodemográficos, as diferenciações no uso e tipos de 


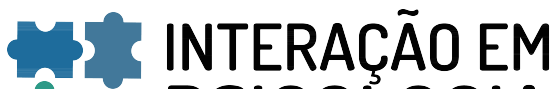 ET PSICOLOGIA}

medicações regulares e participação em outros tratamentos, a compreensão e adequação do procedimento de coleta de dados (no caso da coleta de saliva) também podem ter impactado nesses resultados. Tais aspectos são de difícil controle nas pesquisas envolvendo seres humanos (McLean et al., 2005). Adicionalmente, não foi sistematicamente avaliada a forma como cada participante respondeu ao relaxamento, ainda que na maior parte das sessões elas tenham relatado "ter conseguido relaxar". Em pesquisas futuras poderão ser empregadas tanto medidas de autorrelato (e.g: Relaxation Inventory - Crist, Rickard, PrenticeDunn, \& Barker, 1989) quanto medidas diretas para avaliação de respostas motoras (e.g.: ombros e queixo soltos) ou fisiológicas (e.g.: respiração e batimentos cardíacos lentos) que indiquem os efeitos do relaxamento imediatamente após o treino, minimizando a possiblidade de vieses (Schilling \& Poppen, 1983).

Em síntese, este estudo fornece evidências de que ambos os tratamentos são efetivos para melhorar condições de saúde de mulheres com FM. No entanto, a intervenção integral (intervenção para dor e sono, incluindo o treino de relaxamento) indicou ser mais efetiva, sobretudo na melhora da ansiedade e capacidade funcional. Novos estudos precisam dar continuidade à investigação dos efeitos do treino do relaxamento em comparação às intervenções comportamentais, ampliando-se a amostra e o número de sessões. Um aspecto positivo é que todas as participantes se mantiveram até o final da coleta de dados e realizaram a maior parte das avaliações (apenas 2,3\% de todas as avaliações foram perdidas). Embora se discuta o alto índice de abandono e/ou a desistência de pessoas com dor crônica nos tratamentos de saúde (Kerns et al., 2014), aspectos como este foram contornados com algumas estratégias, como a disponibilidade de um auxiliar de pesquisa para esclarecer dúvidas e buscar as avaliações (coleta da saliva) feitas na casa da participante, disponibilidade de horários e locais para as sessões mais acessíveis a cada participante. Por um cuidado ético, todas as participantes, cujas medidas identificaram mudanças negativas, foram convidadas a participar de atendimentos individuais no Serviço Escola de Psicologia de uma das universidades participantes, imediatamente após o término deste estudo.

\section{CONTRIBUIÇÃO DE CADA AUTOR}

L.F.K. e M.J.D.R. realizaram a conceitualização e metodologia, L.F.K. foi responsável pela análise formal dos dados, administração do projeto, investigação, redação e tabulação dos dados, sob supervisão de M.J.D.R.

\section{Luziane de Fátima Kirchner e Maria de Jesus Dutra dos Reis}

\section{DECLARAÇÃO DE CONFLITOS DE INTERESSES}

Os autores declaram que não há conflitos de interesse no presente artigo.

\section{DECLARAÇÃO DE FINANCIAMENTO}

Coordenação de Aperfeiçoamento de Pessoal de Nível Superior (CAPES).

\section{AGRADECIMENTOS}

Agradecemos ao Dr. Rimenez Rodrigues de Souza pelo auxílio com a análise e descrição da resposta do cortisol.

\section{REFERÊNCIAS}

Bernik, M., Sampaio, T. P., \& Gandarela, L. (2013). Fibromyalgia comorbid with anxiety disorders and depression: Combined medical and psychological treatment. Current Pain and Headache Reports, 17(9), 358. https://doi.org/10.1007/s11916-013-0358-3

Bertolazzi, A. N. (2008). Tradução, adaptação cultural e validação de dois instrumentos de avaliação do sono Dissertação de Mestrado, Universidade Federal do Rio Grande do Sul.

Campbell, D. T., \& Stanley, J. C. (1979). Delineamentos experimentais e quase experimentais de pesquisa. ( $1^{\mathrm{a}}$ ed., R. A. T. Di Dio, Trad.). São Paulo: EPU

Catley, D., Kaell, A. T., Kirschbaum, C., \& Stone, A. A. (2000). A naturalistic evaluation of cortisol secretion in persons with fibrmyalgia and rheumatoid arthritis. Arthritis Care \& Research, 13, 51-61. https://doi.org/10.1002/15290131(200002)13:1

Coppieters, I., Cagnie, B., Nijs, J., Van Oosterwijck, J., Danneels, L., De Pauw, R., \& Meeus, M. (2016). Effects of Stress and relaxation on central pain modulation in chronic whiplash and fibromyalgia patients compared to healthy controls. Pain Physician. 19(3), 119-130.

Crist D. A., Rickard H. C., Prentice-Dunn S., \& Barker H. (1989). The relaxation inventory: Self-report scales of relaxation training effects. Journal of Personality Assessment, 53, 716-726. https://doi.org/10.1207/s15327752jpa5304_8

Cunha, J. A. (2001). Manual da versão em português das Escalas Beck. São Paulo: Casa do Psicólogo.

Fordyce, W. (1976). Behavioral methods for chronic pain and illness. Saint.Louis: Mosby.

Glombiewski, J. A., Sawyer, A. T, Gutermann, J, Koenig, K, Rief, W, \& Hofmann, S. G. (2010). Psychological treatments for fibromyalgia: A meta-analysis. Pain, 151(2), 280-295. https://doi.org/10.1016/j.pain.2010.06.011 


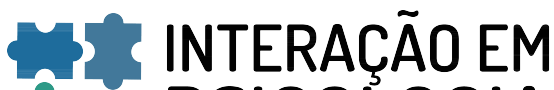 ET PSICOLOGIA}

Gur, A., Karakoc, M., Erdogan, S., Nas, K., Cevik, R, \& Sarac, A. J. (2002). Regional cerebral blood flow and cytokines in young females with fibromyalgia. Clinical and Experimental Rheumatology, 20(6), 753-760. https://doi.org/ 10.1002/1529-0131

Heymann, R. E., Paiva, E., Helfenstein Jr, M., Pollak, D. F., Martinez, J. E., Provenza, J. R. et al. (2010) Consenso brasileiro do tratamento de fibromialgia. Revista Brasileira de Reumatologia, 50 (1), 56-66. http://dx.doi.org/10.1590/ S0482-50042010000100006

Keel, P. J., Bodoky, C., Gerhard, U., \& Muller, W. (1998) Comparison of integrated group therapy and group relaxation training for fibromyalgia. Clinical Journal of Pain, 14(3), 232-238. https://doi.org/10.1097/00002508199809000-00010

Kerns, R. D., Burns, J. W., Shulman, M., Jensen,M. P., Nielson, W. R., Czlapinski, R., \& Dallas, M. I. (2014). Can we improve cognitive-behavioral therapy for chronic back pain treatment engagement and adherence? A controlled trial of tailored versus standard therapy. Health Psychology, 33(9), 938-947. https://doi.org/10.1037/a0034406

Krakow, B. (2006). Potential impact of sleep disorder treatment in fibromyalgia patients. Archives of Internal Medicine, 166(12), 1323-1324. https://doi.org/10.1001/ archinte.166.12.1323-a

Kutz, I., Caudill, M., \& Benson, H. (1983). The role of relaxation in behavioral therapies for chronic pain. International Anesthesiology Clinics, 21(4), 193-200.

Landis, J. R., \& Koch, G. G. (1977). The measurement of observer agreement for categorical data. Biometrics, 33(1), 159-174. https://doi.org/10.2307/2529310

Linton, S. J., \& Götestan, K. G. (1984). A controlled study of the effects of applied relaxation and applied relaxation plus operant procedures in the regulation of chronic pain. British Journal of Clinical Psychology, 23, 291-299. https:// doi.org/10.1111/j.2044-8260.1984.tb01303.x

Lipp, M. E. N. (1997). Relaxamento para todos. Campinas: Papirus Editora.

Lipp, M.E.N. (2000). Inventário de sintomas do stress para adultos. São Paulo: Casa do Psicólogo.

Lundervold, D. A., Talley, C., \& Buermann, M. (2006). Effect of behavioral activation treatment on fibromyalgia - Related pain anxiety cognition. Journal of Behavioral Consultation and Therapy, 2(1), 73-84. https://doi.org/10.1037/ h0100768

Marques A. P., Assumpção, A., Santos, A. M. B., Matsutani, L. A., Lage, L. V., \& Pereira, C. A. B. (2006). Validação da versão brasileira do fibromyalgia impact questionnarie. Revista Brasileira de Reumatologia, 46(1), 24-31. https:// doi.org/10.1590/S0482-50042006000100006
McLean, S. A, Williams, D. A, Harris, R. E, Kop, W. J, Groner, K. $\mathrm{H}$, Ambrose, K, et al. (2005). Momentary relationship between cortisol secretion and symptoms in patients with fibromyalgia. Arthritis \& Rheumatism, 52(11), 3660-3669. https://doi.org/10.1002/art.21372

Meeus, M., Nijs, J., Vanderheiden, T., Baert, I., Descheemaeker, F., \& Struyf, F. (2015). The effect of relaxation therapy on autonomic functioning, symptoms and daily functioning, in patients with chronic fatigue syndrome or fibromyalgia: A systematic review. Clinical Rehabilitation, 29(3), 221 -233. https://doi.org/10.1177/0269215514542635

Melzack, R. (1987). The short-form McGill Pain questionnaire. Pain, 30, 191-197. https://doi.org/0.1016/03043959(87)91074-8

Menzies, V., Lyon, D. E., Elswick, R. K., McCain, N. L., \& Gray, D. P. (2014). Effects of guided imagery on biobehavioral factors in women with fibromyalgia. Journal of Behavioral Medicine, 37(1), 70-80. https://doi.org/10.1007/s10865012-9464-7

NIH Technology Assessment Panel (1996). NIH technology assessment panel on integration of behavioral and relaxation approaches into the treatment of chronic pain and insomnia. JAMA, 276(4), 313-318. https://doi.org/ 10.1001/jama.1996.03540040057033

Perlis, M.L., Jungquist, C., Smith, M.T., Posner, D.(2005) Cognitive Behavioral Treatment of Insomnia. A Session-bySession Guide. New York: Springer.

Provenza, J. R., Pollak, D. F., Martinez, J. E., Paiva, E. S., Helfenstein, M., Heymann, R., Matos, J. M. C., \& Souza, E. J. R. (2004). Diretrizes da fibromialgia - Sociedade Brasileira de Reumatologia. https://doi.org/10.1590/S048250042004000600008

Riedel, W., Layka, H., \& Neeck, G. (1998). Secretory pattern of $\mathrm{GH}, \mathrm{TSH}$, thyroid hormones, ACTH, cortisol, FSH, and $\mathrm{LH}$ in patients with fibromyalgia syndrome following systemic injection of the relevant hypothalamic-releasing hormones. Zeitschrift für Rheumatologie, 57 (2), 81-87. https://doi.org/ $10.1007 / \mathrm{s} 003930050242$

Rimm, D. C., \& Masters, J. C. (1983). Terapia Comportamental: técnicas e resultados experimentais. ( $2^{\mathrm{a}}$ ed., A.F. RodriguesAlves, Trad.). São Paulo: Manole.

Riva, R., Mork, P. J., Westgaard, R. H., Ro, M., \& Lundberg, U. (2010). Fibromyalgia syndrome is associated with hypocortisolism. International Journal of Behavioral Medicine, 17(3), 223-233. https://doi.org/10.1007/s12529010-9097-6

Ross, R. L., Jones, K. D., Bennet, R. M., Ward, R. L., Druker, B. J., \& Wood, L. J. (2010). Preliminary evidence of increased pain and elevated cytokines in fibromyalgia patients with defective growth hormone response to exercise. Open Journal of Immunology, 3, 9-18. https://doi.org/ $10.2174 / 1874226201003010009$ 


\section{-4: INTERACÃO EM IT PSICOLOGIA}

Salvetti, M. G. Pimenta, C. A. M., Braga, P. E., \& Corrêa, C. F. (2012). Incapacidade relacionada à dor lombar crônica: Prevalência e fatores associados. Revista da Escola de Enfermagem da USP, 46, 16-23. https://doi.org/10.1590/ S0080-S62342012000700003

Schilling, D., \& Poppen, R. (1983). Behavioral relaxation training and assessment. Journal of Behavior Therapy and Experimental Psychiatry, 16, 309-316. https://doi.org/ 10.1016/0005-7916(83)90027-7

Tang, N. K. Y., Goodchild, C. E., \& Salkovskis, P. M. (2012). Hybrid cognitive-behaviour therapy for individuals with insomnia and chronic pain: A pilot randomised controlled Trial. Behaviour Research and Therapy, 50(12), 814-821. https://doi.org/10.1016/j.brat.2012.08.006

Thieme, K., \& Turk, D. C. (2005). Heterogeneity of psychophysiological stress responses in fibromyalgia syndrome patients. Arthritis Research \& Therapy, 8(1): R9. https://doi.org/10.1186/ar1863

Turner, J. A. (1982). Comparison of group progressiverelaxation training and cognitive-behavioral group therapy for chronic low back pain. Journal of Consulting and Clinical Psychology, 50(5), 757-765. https://doi.org/ 10.1037/0022-006X.50.5.757

Van Houdenhove, B. \& Luyten P. (2006). Stress, depression and fibromyalgia. Acta Neurologica Belgica, 106(4), 149156.

Vandenberghe, L (2014). Dor- visão comportamental. Em A. Portnoi. A Psicologia da Dor (pp. 15-31). Rio de Janeiro: Guanabara Koogan.

Vera, M. N., \& Vila, J. (2002). Técnicas de relaxamento. Em V. E. Caballo (Org.), Manual de Técnicas de Terapia e Modificação do Comportamento. (2 ed.) (pp. 147-165). São Paulo: Santos Editora.
Vitiello, M. V.. Mccurry, S. M., Shortreed, S. M., Balderson, B. H., Baker, L. D., Keefe F. J., Rybarczyk, B. D., \& Von Korff, M. (2013). Cognitive-behavioral treatment for comorbid insomnia and osteoarthritis pain in primary care: The lifestyles randomized controlled trial. American geriatrics society, 61(6), 947-956. https://doi.org/10.1111/jgs.12275

Wagner, J. S., Chandran, A., DiBonaventura, M., \& Cappelleri, J. C. (2013). The costs associated with sleep symptoms among patients with fibromyalgia. Expert the costs associated with sleep symptoms among patients with fibromyalgia. Pharmacoeconomics \& Outcomes Research, 13(1), 131-139. https://doi.org/10.1586/erp.12.82

Wang, H., Chen, B., \& Chow, S. C. (2003). Sample size determination based on rank tests in clinical trials. Journal of Biopharmaceutical Statistics, 13(40), 735-751. https:// doi.org/10.1081/BIP-120024206

Wegener, S. T., Wolfman, J., \& Haythornthwaite, J. A. (2011). Psychological interventions for chronic pain. In H. Benzon, S. R. Raja, S. E Fishman, S. S. Liu, S. P Cohen (Eds.), Essentials of Pain Medicine. ( $3^{a}$ ed.) (pp. 180-183). Philadelphia: Elsevier Saunders.

Wolpe, J. (1978) Prática da Terapia Comportamental. (2aed. W.G. Clark Jr., Trad.). São Paulo: Brasiliense.

Recebido: em 17/05/2017 Primeira decisão editorial em: 25/07/2017 Aceito em: 16/10/2017 